\title{
Application of PLC in Computer Numerical Control Machine
}

\author{
Shuqin $\mathrm{Xu}$ \\ Department of Transmission and Transformation Technology, \\ Northeast Dianli University, \\ Jilin, Jilin 132012, China
}

\begin{abstract}
At present, in the industrial production, have a high request for the reliability of the control equipment, to be able to in the electromagnetic interference, power voltage fluctuation, mechanical vibration and so on under the harsh environment and reliable work, and has strong anti-jamming ability. PLC is designed for applications in industrial environments designed electronic digital computing system operations, and take a multi-level hardware and software anti-jamming measures in the design and manufacturing process. PLC is widely used in CNC machine and other industrial control, including the continuous control of each axis position, control of spindle positive inversion and spindle rotation on/off, tool changer, clamping and loosening, cooling, chip and other auxiliary action. Modern CNC machine adopts PLC instead of relay control to complete logic control, make the CNC machine tool structure is more compact, more feature-rich, greatly improve the response speed and reliability.
\end{abstract}

Keywords-PLC; computer numerical control machine; CNC; ladder diagram

\section{INTRODUCTION}

PLC is short for programmable logic controller, which combines computer technology, automatic control technology and communication techniques, is made especially for industrial control computer system. In order to meet the requirements of the PLC sequence control, saving some digital operation function of the microcomputer, and strengthen the logic control function. To realize the automatic control device of PLC between the relay control and computer control, but also overcomes some disadvantages of microcomputer, can be used in harsh industrial environments, has strong anti interference ability. In addition to input/output part adopts photoelectric isolation measures, to power, computing, controllers, memory, also set a wide variety of protection and shielding. PLC uses software to realize the user control logic, compact structure, small volume, easy to load the machine internal or electrical box, easy to mechanical and electrical integration of action to achieve complex control logic and CNC machine. At present, most of the PLC adopts and relay logic control circuit diagram is very similar to the ladder diagram of program design, working principle of the graphic symbol image intuitive and easy to understand and master, programming is simple, convenient operation, flexible change procedures. PLC can also be connected to programmer, personal computer, etc, and easily implement program of display, edit, diagnosis, storage and delivery etc. Its working principle is shown in figure 1. 


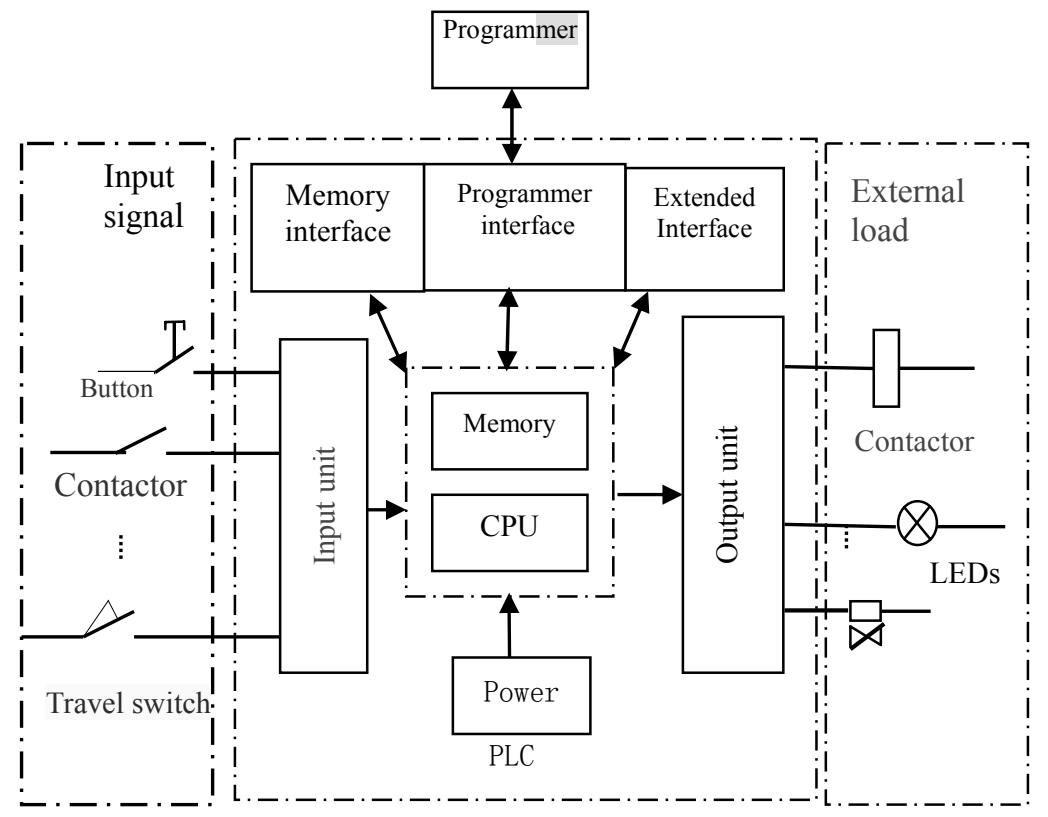

Figure 1. PLC basic composition and working principle

\section{PLC IS COMMONLY USED IN CNC MACHINE TOOL}

PLC in NC machine comes in two forms: built-in type and independent type.

(1) Built-in type: Built in type PLC belongs to the CNC device, signal between PLC and CNC device to transmit on CNC device. Between PLC and CNC machine tool through the $\mathrm{CNC}$ input / output interface circuit for signal transmission.

(2) Independent type: Independent PLC is also called external or general PLC. On the NC machine tool, the independent type PLC is independent of the CNC device, with the hardware structure and the software functions, can complete the control by independent.

\section{CNC MACHINE TOOL, THE PLC CAN REALIZE THE FUNCTION}

CNC machine tools in the design of PLC system and the design of the CNC system is inseparable, the machine numerical control system is generally take or provide the function of PLC.

(1) CNC machine tools using a variety of switch quantity control the movement of the machine tool, including the control switch, trip switch, close to switch, pressure switch, temperature switch, etc., through all kinds of switch signal to the PLC, and after PLC logic operations, to control the signal output to the object.

(2) PLC output signal via the relay, contactor, hydraulic or pneumatic solenoid valve, control knife library manipulator and rotary center workbench, etc.

(3) PLC collects all kinds of fault signal, the warning signs in the appropriate position, issued by the CNC system alarm or alarm text signal.

(4) PLC is to achieve CNC machine tools (MT) of switch control through the communication between the CNC system(CNC) and the machine tool (MT), namely the exchange of information between PLC and CNC, PLC and MT.

\section{INFORMATION INTERCHANGE FOR CNC MACHINE TOOLS}

Information interchange for $\mathrm{CNC}$ machine tool as shown in Figure 2, and mainly consists of the following four parts:

(1) MT $\rightarrow$ PLC, mainly a variety of switches, buttons and other information on the machine operator's panel, Including the machine start and stop, work mode, rate, spindle positive inversion and stop cutting fluid, the opening and closing, chuck and loosen, each coordinate point control, ATC instructions, the over travel limit, spindle servo protection monitoring signal, feed system for signal switch signal. Switch signal machine side can be entered into the PLC by switch of PLC input interface, the $\mathrm{X}$ signal in Figure 2, except for a handful of signal, the meaning of the vast majority of the signal and the PLC occupied address can be defined by the PLC program design. 


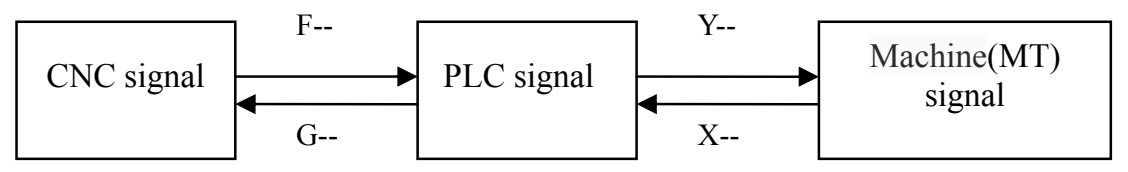

Figure 2. Information transmission between the CNC, PLC and MT

(2) PLC $\rightarrow$ MT, PLC to machine signal control machine actuator, such as solenoid valve, relay, contactor, and ensure the machine all the moving parts of the signal and fault indicator, etc. Signal PLC to control the machine are delivered through a PLC switch output interface to MT, the Y signal in Figure 2, all switch output signals and the meaning of the PLC address are occupied address can be defined by the PLC program design.

(3) $\mathrm{CNC} \rightarrow$ PLC, mainly refers to the function code $\mathrm{M}$, $\mathrm{S}, \mathrm{T}, \mathrm{F}$ etc. S-function by converting the output spindle speed control instructions in PLC; T-function is managed through the PLC tool storage, for automatic tool exchange; M-function depending on the $\mathrm{m}$ code control spindle, reverse, or stop, the gearshift of spindle gear box speed, opening and closing of the cutting fluids, chuck clamping, loosen the tool change manipulator and machining center tool and other actions; F-function to complete the output axis feed rate is controlled by PLC servo system. CNC to the PLC information is available (for CNC side) done through the switch output signal, can also be delivered directly to the PLC registers, the F signal in Figure 2, All of the CNC to the PLC signal meaning and address (switching address and register address) have been determined by CNC manufacturers, PLC programmers can use, but can not be changed or deleted.

(4) PLC $\rightarrow \mathrm{CNC}$, Information of PLC to CNC mainly $\mathrm{M}, \mathrm{S}, \mathrm{T}, \mathrm{F}$ functional response signals and the axis of reference signals, moving parts of machine tools status and fault information. PLC to $\mathrm{CNC}$ information completed by the switching input signals, the $\mathrm{G}$ signal in Figure 2, All of the PLC to the CNC signal meaning and address (switching address and register address) have been determined by CNC manufacturers, PLC programmers can use, but can not be changed or deleted.

Different ways of information exchange between CNC and PLC functions are very different, but its most basic function is the $\mathrm{CNC}$ will be required for the implementation of the $\mathrm{M}, \mathrm{S}, \mathrm{T}, \mathrm{F}$ function codes sent to PLC, then PLC control to complete the corresponding action, PLC signal flow diagram for CNC machine tools in Figure 3. 


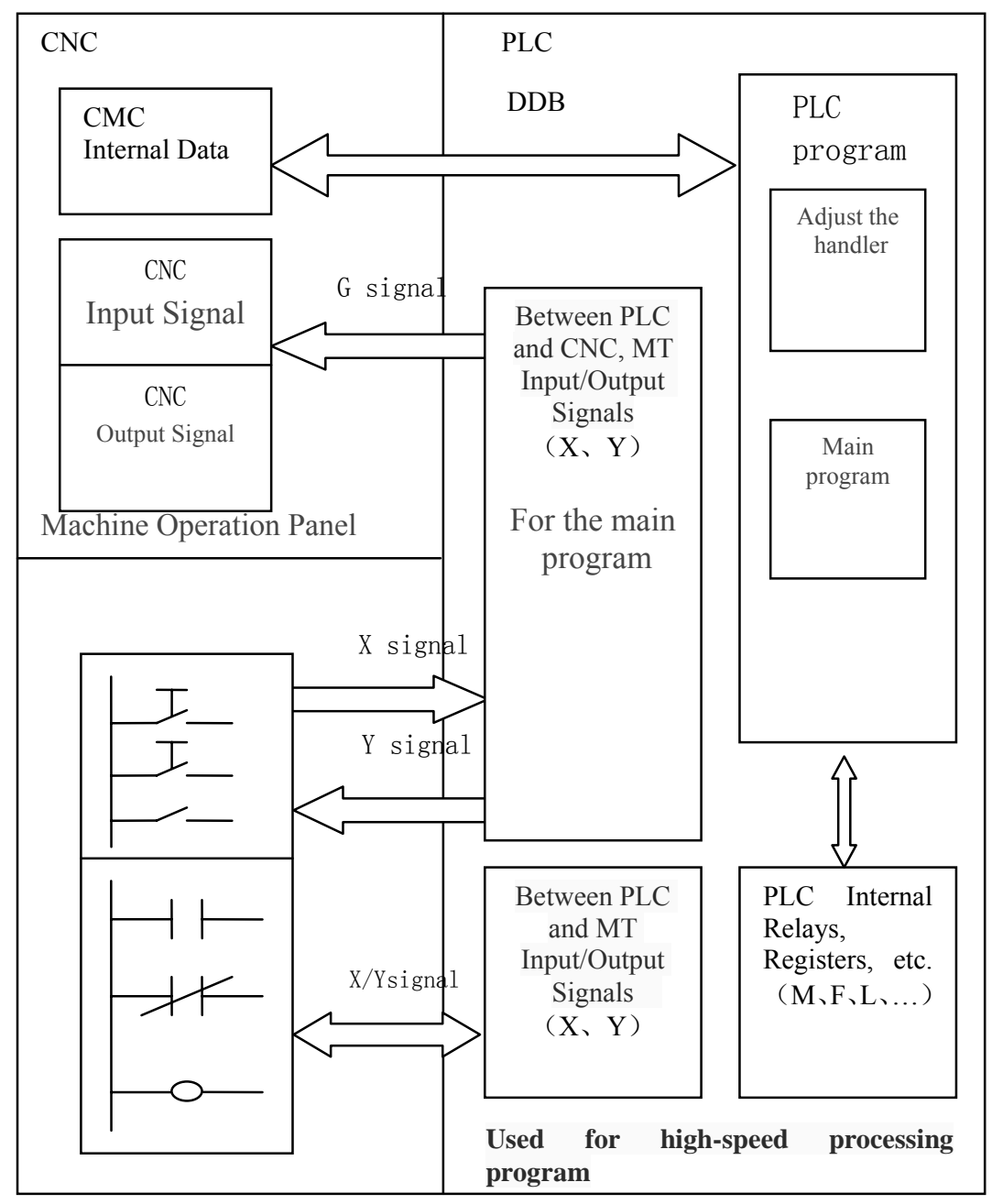

Figure 3. PLC signal flow diagrams

\section{LADDER DIAGRAM SETTING FAILURE DETECTION}

Because the PLC has high reliability, so most of the faults in the PLC control system is not from the PLC itself, but rather caused by external components fault. Once the system detects a component failure, not only with sound and light alarm function, and can immediately display the fault code, so that users can quickly determine the cause of the failure. PLC program not only can detect each fault and displays also judged most critical initial faults automatically. Figure 4 is a ladder diagram with 3 fault detection as an example.

The program is made up of 3 fault detection, respectively $\mathrm{R} 500.0, \mathrm{R} 510.0, \mathrm{R} 520.0 ; 3$ initial fault detection for R500.2, R510.2, R520.2; F149.1 is a system reset signal. The initial state, no alarm, fault detection are " 0 ", and the reset signal F149.1 is "0". 


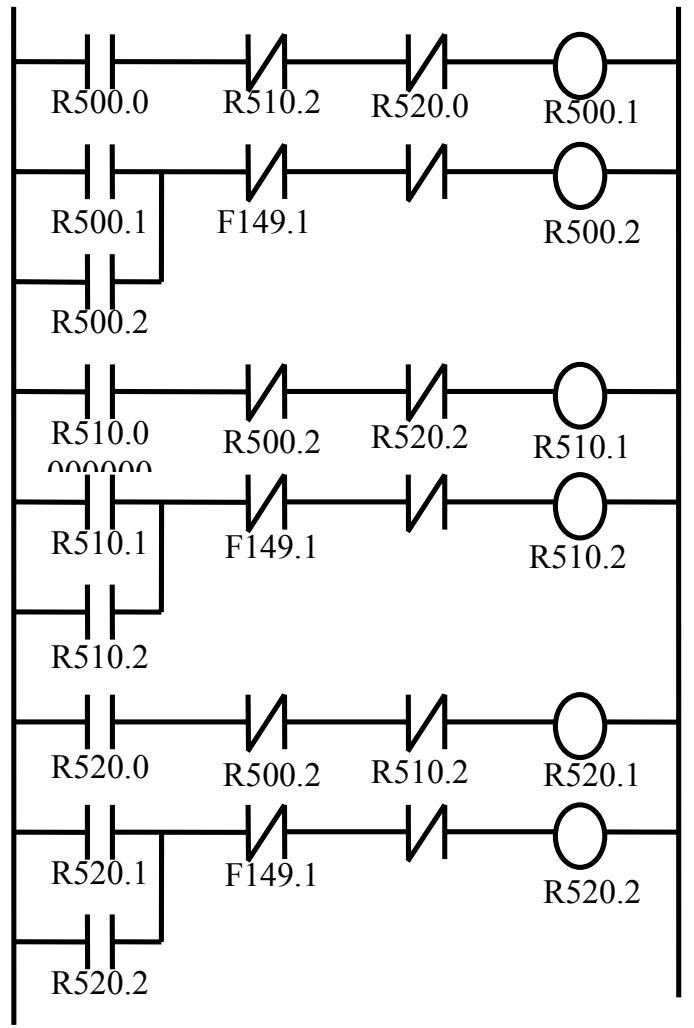

Figure 4. Initial faults of PLC program

\section{CONCLUSION}

With the development of automation technology, the structure and properties of PLC are also constantly improving, using range expanded rapidly, From the overall structure to the development of small modular structure, increasing the flexibility of PLC configuration, PLC computing, data processing, graphics display, network communication and other functions greatly enhanced ,PLC programming and programming tools are also constantly develop towards standardization and senior, so it also can be used more widely in the application of numerical control technology, control of machining center will be more flexible and more reliable.

\section{REFERENCES}

[1] Luo Liangling, Liu xubo. Numerical control technology and its application. Beijing: Qinghua University Press, 2005

[2] Lian Yuanguo, Zhang Yonghong. Design and application of machining center. Beijing: China machine press, 1995

[3] Liao Xiaoguo. CNC technology. Hubei science and technology press, 2000

[4] Zhang Yongle. Programmable controller application. China electric power press, 2004

[5] Gong Shuzhen, Wang dongqing, Xu Shi Xu. Principle and application of programmable logic controller. Beijing posts and telecommunications Publishing Press, 2003

[6] Yu Hanqi, and Guo Jian. Principle and application of programmable logic controller in Beijing: China electric power press, 2004 